\title{
Affine maximal torus fibrations of a compact Lie group
}

\author{
Marcos Salvai* \\ FaMAF - CIEM \\ Ciudad Universitaria, 5000 Córdoba, Argentina
}

\begin{abstract}
By a generalization of the method developed by Gluck and Warner to characterize the oriented great circle fibrations of the three-sphere, we give, for any compact connected semisimple Lie group $G$, a general procedure to obtain the continuous fibrations of $G$ by Weyl-oriented affine maximal tori, find conditions for smoothness and provide infinite dimensional spaces of concrete examples.
\end{abstract}

2000 Mathematics Subject Classification: 55R15, 22E15, 53C12

\section{Introduction}

Let $G$ be a compact connected semisimple Lie group. A maximal torus in $G$ is a maximal abelian Lie subgroup of $G$. A subset $S$ of $G$ is an affine maximal torus if there exist $g, h \in G$ such that $g S h^{-1}$ is a maximal torus of $G$. Equivalently, it is a maximal connected totally geodesic flat submanifold of $G$, provided that the group is endowed with a bi-invariant Riemannian metric.

The problem we deal with in this article is, roughly, in which manners (other than the obvious left- or right-invariant ones) $G$ can be expressed as a disjoint union of affine maximal tori. In the spirit of Gluck and Warner

*Partially supported by CONICOR, CIEM (CONICET) and SECYT (UNC) 
[2], who considered oriented great circle fibrations of the three-sphere, we will study fibrations of $G$ by affine maximal tori which are oriented in the sense of Weyl (see below). That will make our first definitions look rather complicated, but the statements of the results and their proofs will be simpler. Throughout the paper, smooth means of class $C^{\infty}$.

Let $G$ be a compact connected simple Lie group endowed with a biinvariant Riemannian metric. Ranjan proved in [6] that a smooth fibration of $G$ by affine maximal tori induces on the space $\mathcal{F}$ of fibers a Riemannian structure (i.e., $\mathcal{F}$ has a Riemannian structure such that the natural projection $G \rightarrow \mathcal{F}$ is a Riemannian submersion) if and only if the fibration is leftor right-invariant. For $G=S^{3}$, Baird and Wood [1] used techniques similar to those in [2] to obtain a stronger result: only the left- or right-invariant fibrations induce on the space of fibers a conformal structure, or, equivalently, in their terminology, the great circle fibration of $S^{3}$ associated with a conformal morphism from $S^{3}$ to a surface is a Hopf fibration. Perhaps this article can provide some elements which help understand conformal morphisms defined on a compact simple Lie group.

I would like to thank Antonio Di Scala and Carlos Olmos for helpful suggestions.

\section{W-oriented affine maximal torus fibrations}

Let $G$ be as in the introduction a compact connected semisimple Lie group. A tangent vector to $G$ is said to be regular if it is tangent to a unique affine maximal torus. Let $\mathcal{R}$ denote the set of regular tangent vectors. An affine Weyl chamber is a connected component of $\mathcal{R} \cap T_{p} S$, where $S$ is an affine maximal torus and $p \in S$. Given an affine Weyl chamber $C$, there exists a unique affine maximal torus $S$ such that $C$ is contained in $T S$. We denote such a torus by $\tau(C)$. Let $\mathcal{C}$ denote the set of all affine Weyl chambers of $G$.

Fix a maximal torus $T$ and a Weyl chamber $C_{0} \subset T_{e} T . G \times G$ acts transitively on $\mathcal{C}$ on the left as follows: $(g, h) C=d L_{g} d R_{h^{-1}} C$, where $L_{k}, R_{k}$ denote left and right multiplication by $k$, respectively. Since the isotropy subgroup at $C_{0}$ is $\Delta(T)=\{(u, u) \mid u \in T\}, \mathcal{C}$ may be identified with $(G \times G) / \Delta(T)$ and there is a natural bundle structure $\pi: \mathcal{C} \rightarrow G$ defined by $\pi(g, h) \Delta(T)=$ $g h^{-1}$ (in particular, $\left.C \subset T_{\pi(C)} G\right)$.

A Weyl-oriented (briefly, a W-oriented) affine maximal torus is a pair $(S, \rho)$, where $S$ is an affine maximal torus of $G$ and $\rho: S \rightarrow \mathcal{C}$ is a continuous 
function such that $\rho(k) \subset T_{k} S$ for all $k \in S$. It can be shown that there exist $g, h \in G$ such that $S=g T h^{-1}$ and $\rho\left(g u h^{-1}\right)=(g u, h) C_{0}$ for all $u \in T$.

Recall that a fibration of $S^{3}$ by oriented great circles is given by a unit vector field on $S^{3}$ all of whose integral curves are geodesics. Now, a continuous fibration of $G$ by W-oriented affine maximal tori is given by a continuous section $\sigma: G \rightarrow \mathcal{C}$ such that the (continuous) distribution $p \mapsto T_{p} \tau(\sigma(p))$ is integrable and $\tau(\sigma(p))$ is a leaf of the distribution (hence the maximal connected leaf) through $p$ for all $p \in G$. Notice that if $S$ is a leaf, then $\left[\left.\sigma\right|_{S}\right]$ is a W-orientation for $S$. Given such a foliation, the set $\mathcal{F}$ of leaves (which we may suppose to be equipped with the induced W-orientations) with the quotient topology is a topological manifold and the natural projection $G \rightarrow \mathcal{F}$ is a continuous fibration. If $\sigma$ is smooth, then $\mathcal{F}$ admits a differentiable structure such that the natural projection $G \rightarrow \mathcal{F}$ is a smooth fibration (see e.g. [4, Corollary 4 in p 21], the regularity condition is satisfied since given two fibers, there exists an isometry of $G$ taking one to the other, provided that $G$ carries a bi-invariant metric).

Let $\mathcal{T}$ denote the set of all $\mathrm{W}$-oriented affine maximal tori of $G$. The product $G \times G$ acts transitively on $\mathcal{T}$ as follows:

$$
(g, h)(S, \rho)=\left(g S h^{-1},(g, h) \rho(g, h)^{-1}\right)
$$

$\left(G \times G\right.$ acts transitively on $G$ by $\left.(g, h) k=g k h^{-1}\right)$. Let us define the Worientation $\rho_{0}: T \rightarrow \mathcal{C}$ by $\rho_{0}(u)=d L_{u} C_{0}\left(=d R_{u} C_{0}\right)$. Since the isotropy subgroup at $\left(T, \rho_{0}\right)$ is $T \times T$, then $\mathcal{T}$ may be identified in a natural way with $(G \times G) /(T \times T) \cong(G / T) \times(G / T)$. We consider on $\mathcal{T}$ the differentiable structure induced by this identification.

By convention, the intersection of two $\mathrm{W}$-oriented affine maximal tori is the intersection of the underlying tori. A subset of a manifold is a topological submanifold if it is a topological manifold and the inclusion is continuous.

The next Proposition presents a general method to obtain the fibrations of $G$ by W-oriented affine maximal tori. Their smoothness is discussed in Proposition 2.

Proposition 1 Let $\mathcal{F}$ be a compact topological submanifold included in $\mathcal{T}$ with dimension half the dimension of $\mathcal{T}$, such that $S \cap S^{\prime} \neq \emptyset$ for $S, S^{\prime} \in \mathcal{F}$ only if $S=S^{\prime}$ (it is implicit that their $W$-orientations also coincide). Then there exists a unique continuous fibration of $G$ by $W$-oriented affine maximal tori such that $\mathcal{F}$ is its space of $W$-oriented fibers. 
Proposition 2 Let $\sigma: G \rightarrow \mathcal{C}$ be a continuous section inducing a (continuous) fibration $P: G \rightarrow \mathcal{F}$ of $G$ by $W$-oriented affine maximal tori. Then the inclusion $i: \mathcal{F} \rightarrow \mathcal{T}$ is continuous and the following assertions are true.

(a) If $\sigma$ is smooth (in particular, $\mathcal{F}$ admits a differentiable structure such that $P$ is a smooth fibration), then $i: \mathcal{F} \rightarrow \mathcal{T}$ determines a submanifold.

(b) If $i: \mathcal{F} \rightarrow \mathcal{T}$ determines a smooth submanifold, then $\sigma(G)$ determines a smooth submanifold of $\mathcal{C}$.

Remark. A continuous fibration $P: G \rightarrow \mathcal{F}$ of $G$ by W-oriented affine maximal tori is not necessarily smooth, even if the inclusion $i: \mathcal{F} \rightarrow \mathcal{T}$ determines a smooth submanifold, as it is shown in Theorem 4.

\section{Examples}

Consider on $G / T$ any fixed $G$-invariant Riemannian metric. Given a smooth function $f: M \rightarrow M$, where $M$ is a compact Riemannian manifold, let us denote $|d f|=\sup \{\|d f(X)\| \mid X \in T M$ with $\|X\|=1\}$.

The first part of the next theorem provides a sufficient condition for a subset of $\mathcal{T}$ to be the space of $\mathrm{W}$-oriented fibers of some continuous fibration of $G$ by W-oriented affine maximal tori. For $G=S^{3}$, this condition is also necessary (see [2]). In [7] we study the space of affine maximal tori intersecting a fixed one, with the hope that it could help characterize those fibrations. More concrete examples are provided in Theorem 4 and Proposition 6 .

Theorem 3 For each strictly distance decreasing function $f: G / T \rightarrow G / T$, there exists a unique continuous fibration of $G$ by $W$-oriented affine maximal tori, such that graph $(f) \subset G / T \times G / T \cong \mathcal{T}$ is the space of the $W$-oriented fibers. Moreover, the following assertions are true.

(a) If $f$ is smooth and $|d f|<1$, then the fibration is smooth.

(b) A partial converse of (a) holds: If the fibration is smooth, then $f$ is smooth.

\section{Remarks.}

(a) $f=$ constant $=h_{0} T$ corresponds to the section $\sigma(g)=d L_{g} \operatorname{Ad}\left(h_{0}\right) C_{0}$, which produces the left-invariant fibration with fibers $g T h_{0}^{-1}, g \in G$.

(b) Theorem 3 also holds, with a similar proof, if one substitutes the graph of $f$ with its reflection with respect to the diagonal, i.e. $\{(f(y), y) \mid y \in G / T\}$. 
In this case, $f=$ constant $=g_{0} T$ produces the right-invariant fibration with fibers $g_{0} T h^{-1}, h \in G$.

(c) It is an open problem whether a compact connected simple Lie group $G \neq S^{3}$ admits a fibration such that the set of W-oriented fibers as a subset of $\mathcal{T}$ is not the graph of a function from one factor of $G / T \times G / T$ to the other. If $G$ is not simple, this is sometimes the case: take for example the group $G_{1} \times G_{2}$ with the fibration whose fibers are $(g, e)\left(T_{1} \times T_{2}\right)\left(e, h^{-1}\right)$, where $G_{j}$ is a compact connected simple Lie group and $T_{j}$ is a maximal torus of $G_{j}(j=1,2)$.

(d) Gluck and Warner proved in [2] that the converse of Theorem 3 (a) is true for $G=S^{3}$.

(e) Let $h \in G, c T \in N_{G}(T) / T$, the Weyl group of $G$, with $c \notin T$. The graphs of the functions $f_{1}, f_{2}: G / T \rightarrow G / T$ defined by $f_{1}(g T)=h g T$, $f_{2}(g T)=g c T$ do not yield fibrations of $G$ as in Proposition 1, since they always contain two distinct $\mathrm{W}$-oriented affine maximal tori with nonempty intersection. Indeed, $(T, h T) \cap(k T, h k T)=T h^{-1} \cap k T k^{-1} h^{-1}$ contains the identity for all $k \in G$ and $(T, c T) \cap\left(c T, c^{2} T\right)=T c^{-1} \cap c T c^{-2}=T c^{-1}$.

Suppose that the Lie algebra $\mathfrak{g}$ of $G$ is equipped with an $\operatorname{Ad}(G)$-invariant inner product. Let $\mathfrak{t}$ be the Lie algebra of $T$ and let $\mathfrak{m}$ be the orthogonal complement of $\mathfrak{t}$ in $\mathfrak{g}$, which can be identified in a natural way, as a vector space, with $T_{o}(G / T)$ (here $o=e T$ ). Let $E$ be a regular element of $\mathfrak{t}$. It is well-known that the function $\iota: G / T \rightarrow \mathfrak{g}$ defined by $\iota(g T)=\operatorname{Ad}(g) E$ determines a submanifold. Consider on $G / T$ the induced metric, which is $G$-invariant, and let $d$ denote the associated distance. Let $B$ be a normal strongly convex ball of radius $\delta<1$ around $o$ in $G / T$.

The next theorem provides, for any $G$, concrete infinite dimensional spaces of examples of smooth and continuous nonsmooth fibrations of $G$ as in Theorem 3 .

Suppose aditionally that $[X, E]=Y$ for some unit $X, Y \in \mathfrak{m}$ (existence of such $E, X, Y$ follows from standard arguments using the root system associated with $\mathfrak{g}$, see e.g. [3]). Let $\gamma$ be the geodesic in $G / T$ with $\gamma(0)=o$ and initial velocity $X$. Notice that $\gamma$ has unit speed, since $\|\dot{\gamma}(0)\|=\|d \iota(X)\|=$ $\|[X, E]\|=\|Y\|=1$ and that $\left.\gamma\right|_{(-\delta, \delta)}$ is distance preserving, by the choice of $\delta$. 
Theorem 4 For each smooth strictly distance decreasing function $\lambda: \mathbf{R} \rightarrow$ $(-\delta, \delta)$ with $\lambda(0)=0$, the function $f: G / T \rightarrow G / T$ defined by

$$
f(g T)=\gamma(\lambda(\langle\operatorname{Ad}(g) E, Y\rangle))
$$

is smooth and strictly distance decreasing.

Moreover, if $\left|\lambda^{\prime}\right|<1$ (respectively, $\lambda^{\prime}(0)=1$ ), then $|d f|<1$ (respectively, $|d f|=1)$ and the continuous fibration of $G$ by $W$-oriented affine maximal tori associated with $f$ as in Theorem 3 is smooth (respectively, not smooth).

Corollary 5 For any $G$, the hypothesis $|d f|<1$ in Theorem 3 (a) cannot be dropped.

Next, we give a further concrete example of a strictly distance decreasing transformation of $G / T$.

It is well-known that $\iota(G / T)$ determines an isoparametric submanifold of $\mathfrak{g}$ and $T_{E} \iota(G / T) \cong \mathfrak{m}$ decomposes as a direct sum of the common eigenspaces of the shape operators $A_{\xi}$, for $\xi \in\left(T_{E} \iota(G / T)\right)^{\perp} \cong \mathfrak{t}$ [5]. Let $\mathfrak{a}$ be one such eigenspace and let $\operatorname{Exp}_{E}: T_{E} \iota(G / T) \rightarrow \iota(G / T)$ denote the geodesic exponential.

Proposition 6 If $\mathrm{pr}_{\mathfrak{a}}$ denotes the orthogonal projection of $\mathfrak{g}$ onto $\mathfrak{a}$, then the function

$$
f: \iota(G / T) \rightarrow \iota(G / T), \quad f=\operatorname{Exp}_{E} \circ \delta \mathrm{pr}_{\mathfrak{a}}
$$

is strictly distance decreasing.

\section{Proofs of the statements}

By abuse of notation, we will sometimes omit the W-orientation of a $\mathrm{W}$ oriented affine maximal torus, if it is clear from the context; whenever we write $S=g T h^{-1}$, we assume, unless otherwise stated, that $S$ is equipped with the W-orientation $(g, h) \rho_{0}(g, h)^{-1}$.

Proof of Proposition 1. Let $\left\{U_{i}\right\},\left\{V_{i}\right\}$ be finite open coverings of $\mathcal{F}$ such that $\bar{U}_{i}$ (the closure of $U_{i}$, which is compact) is contained in $V_{i}$ and there exist continuous local sections $\left(g_{i}, h_{i}\right): V_{i} \rightarrow G \times G$ of the bundle defined as 
the pullback of the bundle $G \times G \rightarrow \mathcal{T} \cong(G \times G) /(T \times T)$ by the inclusion $\mathcal{F} \hookrightarrow \mathcal{T}$. Hence,

$$
S=g_{i}(S) T h_{i}(S)^{-1}
$$

for all $S \in V_{i}$. Define $F_{i}: V_{i} \times T \rightarrow G$ by $F_{i}(S, u)=g_{i}(S) u h_{i}(S)^{-1}$. First note that $F_{i}$ is injective. Indeed, if $F_{i}(S, u)=F_{i}\left(S^{\prime}, u^{\prime}\right)$, then by $(1) S \cap S^{\prime} \neq \emptyset$ and hence $S=S^{\prime}$ by hypothesis. Thus, $g_{i}(S)=g_{i}\left(S^{\prime}\right)$ and $h_{i}(S)=h_{i}\left(S^{\prime}\right)$. This implies that $u=u^{\prime}$.

Since $F_{i}$ is continuous and $V_{i} \times T$ and $G$ have the same dimension, then $F_{i}$ is an open mapping onto an open subset of $G$. Now, we have by (1) that

$$
\bigcup_{S \in \mathcal{F}} S=\bigcup_{i} F_{i}\left(V_{i} \times T\right)=\bigcup_{i} F_{i}\left(\bar{U}_{i} \times T\right)
$$

is an open and compact subset of $G$, hence it coincides with $G$.

Next, we define for each $i$ the function $\sigma_{i}$ : Image $\left(F_{i}\right) \rightarrow \mathcal{C}$ by

$$
\sigma_{i}\left(F_{i}(S, u)\right)=\left(g_{i}(S) u, h_{i}(S)\right) C_{0} .
$$

Now, the same arguments we have used to show that $F_{i}$ is injective yield that this defines in fact a continuous section $\sigma: G \rightarrow \mathcal{C}$. Finally, given $k \in G$, then $k=F_{i}(S, u)$ for some $i, S \in \mathcal{F}, u \in T$. One easily checks that $k \in S$, $\operatorname{span} \sigma(k)=T_{k} S$ and the W-orientation of $S$ coincides with $\left.\sigma\right|_{S}$.

The following elementary Lemma will be used repeatedly.

Lemma 7 Let $\Pi: Q \rightarrow B$ be a smooth fiber bundle.

(a) If $\mathcal{F}$ determines a smooth submanifold included in $B$, then $\Pi^{-1} \mathcal{F}$ determines a smooth submanifold of $Q$.

(b) If $\Sigma: B \rightarrow Q$ is a continuous section such that $\Sigma(B)$ determines a smooth submanifold of $Q$, then $\Sigma$ is smooth if and only if for all $q \in \Sigma(B)$ one has

$$
T_{q} \Sigma(B) \cap \operatorname{Ker}\left(d \Pi_{q}\right)=\{0\} .
$$

Proof. (a) Let $\iota: \mathcal{F} \rightarrow B$ be the inclusion and let

$$
\iota^{*} Q=\{(x, q) \in \mathcal{F} \times Q \mid \iota(x)=\Pi(q)\}
$$

be the pullback of $Q$ by $\iota$, with the standard differentiable structure. Then the map $\iota^{*} Q \rightarrow Q,(x, q) \mapsto q$ determines a submanifold of $Q$ with image $\Pi^{-1}(\mathcal{F})$. 
(b) Let us denote $P=\left.\Pi\right|_{\Sigma(B)}: \Sigma(B) \rightarrow B$, which is a smooth bijection. If (2) holds for $q=\Sigma(x)$, then $d P_{\Sigma(x)}: T_{\Sigma(x)} \Sigma(B) \rightarrow T_{x} B$ is a linear isomorphism. By the Inverse Function Theorem, $\Sigma$ is smooth. The converse is obvious.

Let us define $\tilde{\tau}: \mathcal{C} \rightarrow \mathcal{T}$ by $\tilde{\tau}(C)=(\tau(C), \rho)$, where $\rho: \tau(C) \rightarrow \mathcal{C}$ is the unique $\mathrm{W}$-orientation of $\tau(G)$ such that $\rho(\pi(C))=C$. Under the identifications given above of $\mathcal{C}$ and $\mathcal{T}$ with homogeneous spaces, one has $\tilde{\tau}(g, h) \Delta(T)=(g T, h T)$ for all $g, h \in G$.

Proof of Proposition 2. Let $k \in G$ be arbitrary and let $U \subset G$ be a smooth submanifold containing $k$ which is transverse to the fibers and intersects each fiber at most once. Then, $P(U)$ is an open set in $\mathcal{F}$ and $s:=\left(\left.P\right|_{U}\right)^{-1}$ : $P(U) \rightarrow G$ is a continuous local section. We have $\left.i\right|_{P(U)}=i \circ P \circ s=\tilde{\tau} \circ \sigma \circ s$. Hence, $i$ is continuous.

Suppose now that $\sigma$ is smooth. Similar arguments yield that $i$ is smooth. Since $k$ is arbitrary, to prove that $i$ determines a submanifold, it suffices to show that $d i_{P(k)}$ is injective. Let $0 \neq X=d P_{k}(Y) \in T_{P(k)} \mathcal{F}$, where $Y \in T_{k} U$, and let $c: I \rightarrow U$ be a smooth curve defined on the interval $I$, with $c(0)=k$ and $c^{\prime}(0)=Y$. There exists a smooth function $(g, h): I \rightarrow G \times G$ such that $\sigma(c(t))=(g(t), h(t)) \Delta(T)$. In particular, $(i \circ P \circ c)(t)=(g(t) T, h(t) T)$ and $c(t)=\pi(\sigma(c(t)))=g(t) h(t)^{-1}$. We differentiate these expressions at $t=0$ and obtain

$$
\begin{aligned}
d i(X) & =(i \circ P \circ c)^{\prime}(0)=\left(d p\left(g^{\prime}(0)\right), d p\left(h^{\prime}(0)\right)\right) \\
\text { and } \quad Y & =d R_{h_{0}^{-1}} g^{\prime}(0)-d L_{g_{0}} d L_{h_{0}^{-1}} d R_{h_{0}^{-1}} h^{\prime}(0),
\end{aligned}
$$

where $p: G \rightarrow G / T$ denotes the canonical projection and $g_{0}=g(0), h_{0}=$ $h(0)$. The first equation implies that if $d i(X)=(0,0)$, then there exist $Z, Z^{\prime} \in \mathfrak{t}$ such that $g^{\prime}(0)=d L_{g_{0}} Z$ and $h^{\prime}(0)=d L_{h_{0}} Z^{\prime}$. After substitution in the second equation in (3), we have

$$
Y=d L_{g_{0}} d R_{h_{0}^{-1}}\left(Z-Z^{\prime}\right) \in T_{k} \tau \sigma(k) .
$$

This is a contradiction, since $\tau \sigma(k)$ is the fiber through $k$ and $Y \neq 0$ is tangent to a submanifold transverse to the fibers. Therefore, $d i$ is injective and $i$ determines a submanifold. This proves (a). 
Next, we prove (b). By Lemma 7 (a), with $\tilde{\tau}: \mathcal{C} \rightarrow \mathcal{T}$ instead of $\Pi$ : $Q \rightarrow B, \tilde{\tau}^{-1}(\mathcal{F})$ is a smooth submanifold of $\mathcal{C}$. It suffices to show that $\sigma(G)=\tilde{\tau}^{-1}(\mathcal{F})$. Indeed, clearly, $\tilde{\tau} \sigma(G) \subset \mathcal{F}$ and if $\tilde{\tau} C \in \mathcal{F}$, then $C=$ $\sigma(\pi(C)) \in \sigma(G)$.

For $S \in \mathcal{T}$, let $I(S)$ denote the set of all tori in $\mathcal{T}$ whose intersection with $S$ is not empty. If $(M, d)$ is a metric space and $(p, q) \in M \times M$, let $D(p, q)=\left\{\left(p^{\prime}, q^{\prime}\right) \in M \times M \mid d\left(p, p^{\prime}\right)=d\left(q, q^{\prime}\right)\right\}$. If one identifies as above $\mathcal{T}$ with $G / T \times G / T$, then $D(S) \subset G / T \times G / T$ for $S \in \mathcal{T}$.

Lemma $8 I(S) \subset D(S)$ for all $S \in \mathcal{T}$.

Proof. Suppose that $S=(g T, h T)$. First we show that $S^{\prime} \in I(S)$ if and only if $S^{\prime}=(g u k T, h k T)$ for some $u \in T, k \in G$. Indeed, if $S^{\prime}$ has this form, then $g u h^{-1} \in S \cap S^{\prime}$. Conversely, if $S^{\prime}=(\tilde{g} T, \tilde{h} T)$ and $S \cap S^{\prime} \neq \emptyset$, then there exist $u, v \in T$ such that $g u h^{-1}=\tilde{g} v \tilde{h}^{-1}$. Calling $k=h^{-1} \tilde{h}$, one has $\tilde{g} v=g u k$ and $\tilde{h}=h k$, as desired.

Now, if $S^{\prime}=(g u k T, h k T) \in I(S)$ as above, we compute

$$
d(g u k T, g T)=d(u k T, T)=d(k T, T)=d(h k T, h T),
$$

since the metric on $G / T$ is $G$-invariant. Therefore, $S^{\prime} \in D(S)$.

Remark. We prove in [7] that $I(S)=D(S)$ for all $S \in \mathcal{T}$ if and only if $G=S^{3}$.

Proof of Theorem 3. Let $f: G / T \rightarrow G / T$ be a strictly distance decreasing function. We have to check that $\mathcal{F}=\operatorname{graph} f$ satisfies the hypotheses of Proposition 1. Clearly, it is a compact topological submanifold of $G / T \times G / T$ with the required dimension. Now, suppose that $S, S^{\prime} \in \mathcal{F}$ (say, $S=(x, f(x))$ and $\left.S^{\prime}=(y, f(y))\right)$ and $S \cap S^{\prime} \neq \emptyset$, or equivalently, that $S^{\prime} \in I(S)$. Since $I(S) \subset D(S)$ by Lemma 8 , we have that $d(y, x)=d(f(y), f(x))$ and this implies that $S=S^{\prime}$ ( $f$ is strictly distance decreasing).

(a) Suppose additionally that $f$ is smooth and $|d f|<1$ (in particular, graph $f$ is a smooth submanifold of $\mathcal{T}$ ). Let $\sigma: G \rightarrow \mathcal{C}$ be the continuous fibration of $G$ associated with $f$, as above. By Proposition $2(\mathrm{~b}), \mathcal{S}:=\sigma(G)$ determines a smooth submanifold of $\mathcal{C}$. We must prove that $\sigma$ is smooth. By 
Lemma 7 (b), with $\pi: \mathcal{C} \rightarrow G$ instead of $\Pi: Q \rightarrow B$ and $\Sigma=\sigma$, it suffices to show that

$$
T_{C} \mathcal{S} \cap \operatorname{Ker}\left(d \pi_{C}\right)=\{0\}
$$

for all $C \in \mathcal{S}$. If we identify as above $\mathcal{C} \cong(G \times G) / \Delta(T)$ and $C \cong$ $(g, h) \Delta(T)$, then

$$
T_{C} \mathcal{C}=d \tilde{L}_{(g, h)}\left(\mathfrak{m} \oplus \mathfrak{m} \oplus \Delta^{-}(\mathfrak{t})\right),
$$

where $\tilde{L}_{k}$ denotes left multiplication by $k$ in the quotient space and $\Delta^{-}(\mathfrak{t})=$ $\{(Z,-Z) \mid Z \in \mathfrak{t}\}$. Now, we verify that

$$
\operatorname{Ker}\left(d \pi_{C}\right)=\left\{\left(d \tilde{L}_{(g, h)}\right)_{\Delta(T)}(Y, Y) \mid Y \in \mathfrak{m}\right\}
$$

Indeed, given $Y \in \mathfrak{m}$, we compute

$$
d \pi d \tilde{L}_{(g, h)}(Y, Y)=\left.\frac{d}{d t}\right|_{t=0} \pi(g, h)(\exp t Y, \exp t Y) \Delta(T)=\left.\frac{d}{d t}\right|_{t=0} g h^{-1}=0 .
$$

Since $\pi: \mathcal{C} \rightarrow G$ is a submersion and $\operatorname{dim} \mathcal{C}-\operatorname{dim} G=\operatorname{dim} \mathfrak{m},(5)$ follows. Next we compute for $Y \in \mathfrak{m}$,

$$
\begin{aligned}
d \tilde{\tau} d \tilde{L}_{(g, h) \Delta(T)}(Y, Y) & =\left.\frac{d}{d t}\right|_{t=0} \tilde{\tau}(g, h) \exp t(Y, Y) \Delta(T) \\
& =\left.\frac{d}{d t}\right|_{t=0}(g \exp (t Y) T, h \exp (t Y) T) \\
& =\left(d \tilde{L}_{g} Y, d \tilde{L}_{h} Y\right) \in T_{g T}(G / T) \times T_{h T}(G / T)
\end{aligned}
$$

On the other hand, if $d \tilde{L}_{(g, h) \Delta(T)}(Y, Y) \in T_{C} \mathcal{S}$, we have

$$
\left\|d \tilde{L}_{g} Y\right\|=\left\|d \tilde{L}_{h} Y\right\|=\left\|d f_{g T}\left(d \tilde{L}_{g} Y\right)\right\|
$$

since the image of $\tilde{\tau} \circ \sigma$ is graph $(f)$. Therefore, (4) follows from (5) if $|d f|<1$.

(b) Suppose that the section $\sigma: G \rightarrow \mathcal{C}$ associated with $f$ is smooth and let $P: G \rightarrow \operatorname{graph}(f)$ be the corresponding smooth fibration. By Proposition $2($ a), graph $(f)$ determines a smooth submanifold of $\mathcal{T}$. Now, we apply Lemma 7 (b), with $\Pi$ the projection $\operatorname{pr}_{1}$ onto the first factor of $(G / T) \times(G / T)$ 
and $\Sigma(x)=(x, f(x))$, to show that $\Sigma$ (and hence $f$ ) is smooth. We must check that

$$
T_{(x, f(x))} \operatorname{graph}(f) \cap \operatorname{Ker}\left(d \operatorname{pr}_{1}\right)_{(x, f(x))}=\{0\}
$$

for all $x \in G / T$. Take an arbitrary element $(0, Z) \in \operatorname{Ker}\left(d \mathrm{pr}_{1}\right)_{(x, f(x))}$, where $Z \in T_{f(x)}(G / T)$, and suppose that $(0, Z) \in T_{(x, f(x))} \operatorname{graph}(f)$. Let $\gamma(t)=$ $(a(t), f(a(t)))$ be a smooth curve in graph $(f)$ with $\gamma^{\prime}(0)=(0, Z)$. We compute

$$
\begin{aligned}
\|Z\| & =\left\|(f \circ a)^{\prime}(0)\right\|=\lim _{t \rightarrow 0^{+}} \frac{d(f(a(t)), f(a(0)))}{t} \leq \\
& \leq \lim _{t \rightarrow 0^{+}} \frac{d(a(t), a(0))}{t}=\left\|a^{\prime}(0)\right\|=0
\end{aligned}
$$

Hence $Z=0$ and thus (6) holds.

$\diamond$

Proof of Theorem 4. The function $f$ is smooth and strictly distance decreasing, since $f$ is clearly a composition of smooth distance decreasing functions and one of them, $\lambda$, is strictly distance decreasing. Let $Z \in \mathfrak{m}$ with $\|d \iota(Z)\|=\|[Z, E]\|=1$, let $g \in G$ and denote $a(g)=\langle\operatorname{Ad}(g) E, Y\rangle$. We compute

$$
\begin{aligned}
d f_{g T}\left(d \tilde{L}_{g} Z\right) & =\left.\frac{d}{d t}\right|_{t=0} f(g \exp (t Z) T) \\
& =\left.\frac{d}{d t}\right|_{t=0} \gamma(\lambda(\langle\operatorname{Ad}(g \exp t Z) E, Y\rangle)) \\
& =\lambda^{\prime}(a(g))\langle\operatorname{Ad}(g)[Z, E], Y\rangle\left(\gamma^{\prime} \circ \lambda \circ a\right)(g) .
\end{aligned}
$$

If $\left|\lambda^{\prime}\right|<1$, then $|d f|<1$, since $\operatorname{Ad}(g)$ is orthogonal and $\gamma$ is unit speed. Hence, the fibration is smooth by Theorem $3(\mathrm{a})$.

Suppose now that $\lambda^{\prime}(0)=1$. Setting in (7) $g=e$ and $Z=X\left(=\gamma^{\prime}(0) \in\right.$ $\mathfrak{m})$, one obtains

$$
d f_{o}(X)=\lambda^{\prime}(0)\langle[X, E], Y\rangle X=X .
$$

Hence $|d f| \geq 1$ and thus $|d f|=1$, since $f$ is distance decreasing. Let $\sigma$ : $G \rightarrow \mathcal{C}$ be the continuous section associated with $f$ as in Theorem 3. By Proposition $2(\mathrm{~b}), \sigma(G)$ determines a smooth submanifold of $\mathcal{T}$. Identifying as above $\mathcal{C} \cong(G \times G) / \Delta(T)$, one has

$$
d \tilde{\tau}_{\Delta(T)}(X, X)=(X, X)=\left(X, d f_{o} X\right) \in T_{(o, o)}(\operatorname{graph} f) .
$$


Hence, $(X, X) \in T_{\Delta(T)} \sigma(G)$, since $\sigma(G)=\tilde{\tau}^{-1}$ (graph $f$ ). Moreover, by (5), $(X, X) \in \operatorname{Ker}\left(\left.d \pi\right|_{\Delta(T)}\right)$. Therefore, $\sigma$ is not smooth by Lemma 7 (b), with $\pi: \mathcal{C} \rightarrow \mathcal{T}$ instead of $\Pi: Q \rightarrow B$ and $\Sigma=\sigma$.

Proof of Proposition 6. It follows from Toponogov's Theorem and the fact that $\operatorname{Exp}_{E}\left(\mathfrak{m}_{\alpha}\right)$ is a totally geodesic round sphere (see [5]).

\section{References}

[1] P Baird, J C Wood, Bernstein theorems for harmonic morphisms from $\mathbb{R}^{3}$ and $S^{3}$, Math. An. 280 No. 4 (1988), 579-603.

[2] H Gluck, F Warner, Great circle fibrations of the three-sphere, Duke Math. J. 50 (1983), 107-132.

[3] S Helgason, Differential geometry, Lie groups, and symmetric spaces, Academic Press, 1978.

[4] R Palais, A global formulation of the Lie theory of transformations groups, Memoirs A.M.S. No. 22 (1957).

[5] R Palais, C-L Terng, Critical point theory and submanifold geometry, Lecture Notes in Mathematics 1353, Berlin, Heidelberg, New York (1998).

[6] A Ranjan, Riemannian submersions of compact simple Lie groups with connected totally geodesic fibers, Math. Z. 191 No. 2 (1986), 239-246.

[7] M Salvai, Affine maximal tori intersecting a fixed one, to appear in Contemp. Math., Proceedings of the International Congress on Differential Geometry in Memory of Alfred Gray, ed. M Fernández and J Wolf.

Email: salvai@mate.uncor.edu

Fax: ++54-351-4334054 